\title{
Experience with Low-Dose Sodium Valproate in Idiopathic Generalized Epilepsy Patients: A Long-Term Follow-Up Study on Selective Cohort from North India
}

\author{
Deepak Goel ${ }^{1} \quad$ Manish Mittal $^{1}$ \\ ${ }^{1}$ Department of Neurology, Swami Ram Himalayan University, \\ Dehradun, India
}

J Neurosci Rural Pract:2021;12:46-50

\begin{abstract}
Address for correspondence Deepak Goel, MD, DM, Department of Neurology, Swami Ram Himalayan University, Dehradun 248001, India (e-mail: goeld007in@yahoo.co.in).
\end{abstract}

\begin{abstract}
Keywords

- low-dose valproate

- idiopathic generalized epilepsy

- treatment of epilepsy

Background Idiopathic generalized epilepsy (IGE) is found in 20 to $30 \%$ of all patients presenting with seizures. Most of the patients require lifelong drug treatment. Efficacy and tolerability are important issues while selecting the most appropriate drug for a person with IGE.

Objective The aim of this study was to look for usefulness of small dose valproate (<1,000 mg/day) in long-term treatment of IGE patients.

Methods Diagnosis of IGE made with standard criteria among all patients presenting with seizures. Patients put on full doses of valproate $(>1,000 \mathrm{mg} /$ day $)$ in first year, then reduction started in next year in patients with full seizure remission, and finally maintained on lowest possible dose of valproate. Lowest dose was defined as the minimum dose without seizures (between 200 and 900 mg/day). Patients, who were refractory on monotherapy, were put on add-on drug and followed for remission and reduction in doses of valproate at minimum possible dose.

Results IGE was diagnosed in $21 \%$ of all patients presenting with seizures. Among 420 patients of IGE 368 (87.5\%) were started on high-dose valproate monotherapy, 155 (42.1\%) were responsive to single drug while 213 (57.9\%) had been given add-on drug either lamotrigine or clonazepam or both. After minimum 3-year follow-up, $298(81 \%)$ could be managed on low-dose valproate $(<1,000 \mathrm{mg})$ without any relapse during 12 to 80 months follow-up.

Conclusion Significant number of patients with IGE can be managed on low-dose valproate with good seizure control and less side effects.
\end{abstract}

\section{Introduction}

Idiopathic generalized epilepsy (IGE) is a well-recognized electro-clinical syndrome of epilepsy. ${ }^{1,2}$ IGE is affecting all gender and most cases have onset before 20 years of age. $^{2}$ Various epileptic syndromes recognized under the heading of IGE are diagnosed on the basis of characteristic clinical features and electroencephalographic (EEG)

DOI https://doi.org/ 10.1055/s-0040-1718853 ISSN 0976-3147. findings. ${ }^{1}$ Most of the patients respond well to monotherapy but often requires lifelong treatment. ${ }^{3.4}$ Sodium valproate (SVP), levetiracetam (LEV), lamotrigine (LTG), and topiramate (TPM) are most commonly tested antiepileptic drugs for IGE. ${ }^{5-7}$ Perampanel is a new drug and is also being recently tested for IGE treatment successfully. ${ }^{8}$ The important issue related with long duration (may be lifelong) of treatment is

(c) 2021. Association for Helping Neurosurgical Sick People.

This is an open access article published by Thieme under the terms of the Creative Commons Attribution-NonDerivative-NonCommercial-License, permitting copying and reproduction so long as the original work is given appropriate credit. Contents may not be used for commercial purposes, or adapted, remixed, transformed or built upon. (https://creativecommons.org/licenses/by-nc-nd/4.0/)

Thieme Medical and Scientific Publishers Pvt. Ltd., A-12, 2nd Floor, Sector 2, Noida-201301 UP, India 
drug tolerability and long-term adverse effects of selected antiepileptic drug. Overall, with long-term experience and evidences, SVP is the most effective monotherapy in patients of IGE. ${ }^{3-5}$ Many concerns raised with the long-term use of SVP in persons with IGE due to drug-related adverse reactions like weight gain, hair loss, tremors, and additional risk of pregnancy-related complications in female. ${ }^{9}$

It is not appropriate if patients of IGE are deprived from the most effective drug (SVP) due to fear of side effects when the alternative antiepileptic drug might be costlier and can have poor seizure control. Since long-term and pregnancy-related side effects of SVP are dose related, and adverse effects at lower doses of SVP are comparable to other drugs. ${ }^{10}$ Therefore, if a low-dose SVP therapy is successful for persons with IGE, then it can be a balance approach with good effective treatment and better tolerability. One study had already shown that low-dose SVP is highly effective treatment for majority of IGE patients. ${ }^{11}$ We had further analysis on Indian patients suffering from IGE and tried to assess the long-term efficacy of low-dose SVP in patients with IGE.

\section{Methods}

Prospective hospital-based study conducted between 2006 and 2015 form a single tertiary level teaching hospital. All patients presenting with seizures were prospectively evaluated in our department. Detail clinical history, age of onset, age of presentation, type of seizures, brain imaging findings from either computerized tomography (CT) or magnetic resonance image (MRI) scan, and EEG findings were recorded for all patients. Seizure classification was based on to International League Against Epilepsy (ILAE). ${ }^{12}$

Case definition, criteria of diagnosis, and classification of IGE were according to ILAE 1989 classification. ${ }^{1,13}$ Patients of acute symptomatic seizures, focal epilepsy, and epileptic encephalopathy were excluded from the cohort. Patients having age of onset of seizures below 6 years or above 31 years, and having global developmental delay were also excluded. Minor abnormalities on CT/MRI like nonreactive calcified lesion, cerebellar atrophy, and incidental arachnoid cysts were not excluded.

After confirmation of the diagnosis of IGE patients were classified in three broad categories: (1) childhood and juvenile absence epilepsies (CAE/JAE), (2) juvenile myoclonic epilepsy (JME), and (3) IGE with generalized tonic clinic seizure (GTCS) only. After appropriate antiepileptic drug selection, patients were followed for minimum 2 years. Full doses of antiepileptic drugs according weight was continued minimum for 2 years. Patients, who had adverse drug reaction to first antiepileptic drug selected, were shifted to alternate monotherapy. Remission was defined when patient remained seizure free for 2 years after which doses were tried to reduce at small single daily dose. For confirming the seizure remission, both major and minor seizures (myoclonic or absence and unaware tongue bites) were asked from patients and family members. Remission was considered when there were no major and minor seizures in follow-up. Along with clinical remission normalization of EEG at the end of 2 year, treatment was essential for reduction of the doses. Further follow-up of 1 year was done to see for any clinical relapse or reappearance of EEG abnormality at every 3 months interval on small single daily doses. Those patients, who had seizures on first monotherapy, were subjected to add-on antiepileptic drugs. Even in patients on duo-therapy having 2 years successful remission were given a trial of small single daily doses on monotherapy.

Patients of IGE on SVP were our target population. High dose of SVP was described as any dose $>1,000 \mathrm{mg}$ total dose and low dose was defined as $<1,000 \mathrm{mg}$ total dose. All patients were initially maintained on doses of $>20 \mathrm{mg} / \mathrm{kg}$ body weight for at least 1 year and reduction of doses started after successful remission for 1 year to less than $15 \mathrm{mg} / \mathrm{kg}$ body weight and finally to $10 \mathrm{mg} / \mathrm{kg}$ body weight for maintenance at every 3 months interval. Our data were analyzed after 3 years successful follow-up with 1 year of full doses (induction phase), 1 year reducing doses every 3 months (tapering phase), and 1 year low maintenance doses (maintenance phase) of all the patients of IGE on SVP. Any patient having relapse of seizure in tapering phase was again put on previous dose at which remission was there. Low-dose SVP was described in three categories: (1) 200 to 300 , (2) 400 to 600 , and (3) 700 to $900 \mathrm{mg} /$ day. Successful remission in patients on either monotherapy or duo-therapy was calculated. Percentages of patients having persistent remission on less than $\leq 900 \mathrm{mg}$ daily dose of SVP were calculated. Female patients of child-bearing age were supplemented with $5 \mathrm{mg}$ daily folic acid tablet with any antiepileptic given.

A crosstab analysis using IBM SPSS Statistics for Windows version 20 (Armonk NY: IBM Corp.) and p-value was calculated using Chi-square test. Analysis of significance was done between the two groups of patients (those who had relapsed vs. no relapse during treatment). Following variables were compared between the groups: (1) gender, (2) age of onset and presentation, (3) type of IGE, (4) duration of illness, (5) numbers and doses of antiepileptic drugs used, and (6) reason of presentation. A $p$-value of $<0.05$ was counted as significant.

\section{Results}

Total 552 (21\%) among total 2,631 patients of recurrent seizures had clinical and electrographic features of IGE and were on SVP. Among these 552 patients, 32 had onset below 6 years, nine patients had onset above 31 years, 91 could not followed up for 3 years and eight had features of focal seizures (pseudo-absence) in follow-up were excluded from analysis. Finally, total 420 patient of IGE on SVP with full follow-up of 3 years or more were included for final analysis. Our cohort had 189 (45\%) females and 231 (55\%) males population with $18.7( \pm 7.4)$ years mean age of presentation and $12.8( \pm 5.98)$ year age of onset of IGE. Only $41(9.8 \%)$ patients had age more than 20 years at the time of first seizure. Among all 143 (34\%) patients had presented due to new onset illness, $187(44.5 \%)$ presented due to poor control of seizures and $90(21.5 \%)$ presented due to relapse after stopping antiepileptic drugs. Total duration of illness of known cases of IGE was 
ranging from 1 to 32 years (median duration of 5 years). Total 317 (75.5\%) patients had IGE with pure GTCS, 69 (16.4\%) had JME, and $34(8.1 \%)$ had childhood or juvenile absence epilepsies (CAE/JAE). Family history of seizures among first blood relatives was positive in $72(17.1 \%)$ patients of IGE. Total 71 (16.9\%) patients had normal initial EEG and brain imaging done in 394 (93.8\%) patients that showed minor abnormalities in five (1.2\%) patients rest CT/MRI were normal. Total duration of follow-up after 2 years treatment and doses tapering trial was ranging from 12 to 80 months. Profile of all patients on SVP therapy is shown in - Table 1.

Among 420 patients, SVP was less than $1,000 \mathrm{mg} /$ day in 52 (12.4\%) patients according to weight and were not included in analysis. SVP of $>1,000 \mathrm{mg} /$ day was used in total 368 (87.6\%) patients and was initially tolerated well in all. During follow-up of 368 (87.6\%) patients of IGE on SVP, 155 (42.1\%) had remission on monotherapy, while 213 (57.9\%) required add-on therapy due to poor response to monotherapy. Total 127 (34.5\%) were on duo-therapy of SVP + LTG (SVP + LTG), $48(13 \%)$ were on SVP + clonazepam (SVP + CZP), and 38 (10.5\%) on three drugs (SVP + LTG + CZP).

Among 155 (42.1\%) patients who had remission on monotherapy were on $>1,000 \mathrm{mg} /$ day of SVP according to $>20 \mathrm{mg} / \mathrm{kg}$ body weight. After 1 year of complete remission of seizures tapering of SVP was tried, it was successful in $129(83.2 \%)$ patients, while 26 (16.8\%) had early relapse so again continued on high doses of SVP (>1,000 mg/day). Among 129 patients maintained on single low dose of SVP, 22 (17\%) were on 200 to $300 \mathrm{mg} /$ day, 81 (62.8\%) were on 400 to $600 \mathrm{mg} /$ day, and 26 (20.2\%) were 700 to $900 \mathrm{mg} /$ day of maintenance doses. Total 129 patients with monotherapy had mean follow-up of 26.8 months (12-80 months). During long follow-up, 13 (8.4\%) patients had relapses of seizures but still manage with low-dose SVP $(<1,000 \mathrm{mg} /$ day $)$. Finally, $129(83.2 \%)$ were successfully maintained on low doses of SVP without any relapse. Only seven (4.5\%) patients reported adverse reaction on monotherapy (weight gain and tremors).

Total 127 (34.5\%) patients had 1 year remission on combination of SVP + LTG. In this group, we also tried to tapper SVP after 1 year of successful remission and it was achieved in 100 (78.7\%) but failed in $27(21.3 \%)$ and thus continued high doses of SVP. Among 99 patients who had remission on low doses, total 15 (15\%) were on 200 to $300 \mathrm{mg} /$ day, $64(64 \%)$ were on 400 to $600 \mathrm{mg} / \mathrm{day}$, and $21(21 \%)$ were on 700 to $900 \mathrm{mg} /$ day of maintenance doses of SVP along with LTG. Among all 99 patients put on low doses of SVP, 18 had relapse in 1 year follow-up and dose acceleration was done still all 18 patients were on $<1,000 \mathrm{mg}$ daily doses, and thus, finally 99 (78.6\%) had successful remission on low doses of SVP. Total six (4.8\%) patients reported adverse reaction.

Among 86 (23.4\%) patients (SVP + CZP and SVP + LTG + CZP), 17 (20\%) patients could have remission on high doses of SVP and 69 (80\%) were managed on low dose of SVP. Details of distribution of all patients on low-dose SVP therapy is shown in - Table 2.

Overall among 368 patients with IGE 298 (81\%) could be managed with low doses of SVP without any relapse with mean follow-up of 26 months (12-82 months). Although 123
Table 1 Profile of patients with idiopathic generalized epilepsy

\begin{tabular}{|c|c|}
\hline Parameters & Number (\%) \\
\hline $\begin{array}{l}\text { Total patients ( } n \text { ) of IGE on } \\
\text { valproate }\end{array}$ & $420(100)$ \\
\hline Male: female & $231: 189(1.2: 1)$ \\
\hline $\begin{array}{l}\text { Age of presentation, } \\
\text { range (mean) }\end{array}$ & $6-42$ years $(18.7)$ \\
\hline Age of onset, range (mean) & $6-30$ years $(12.8)$ \\
\hline \multicolumn{2}{|l|}{ Reasons for presentation } \\
\hline New onset epilepsy & $143(34)$ \\
\hline Poor control of epilepsy & $187(44.5)$ \\
\hline $\begin{array}{l}\text { Relapse after drug } \\
\text { withdrawal }\end{array}$ & $90(21.5)$ \\
\hline \multicolumn{2}{|l|}{ Type of IGE } \\
\hline IGE with pure GTCS & $317(75.5)$ \\
\hline $\begin{array}{l}\text { Juvenile myoclonic } \\
\text { epilepsy }\end{array}$ & $69(16.4)$ \\
\hline Absence epilepsy & $34(8.1)$ \\
\hline Positive family history & $72(17.1)$ \\
\hline \multicolumn{2}{|l|}{ First drug used } \\
\hline $\begin{array}{l}\text { SVP }>1,000 \mathrm{mg} / \text { day to } \\
\text { start }\end{array}$ & $368(87.6)$ \\
\hline $\begin{array}{l}\text { Other than SVP } \\
<1,000 \mathrm{mg} / \text { day to start }\end{array}$ & $52(12.4)$ \\
\hline \multicolumn{2}{|c|}{ Number of antiepileptic drugs used } \\
\hline Monotherapy (only SVP) & $155(42.1)$ \\
\hline Add-on therapy & $213(57.9)$ \\
\hline
\end{tabular}

Abbreviations: GTCS, generalized tonic-clonic seizure; IGE, idiopathic generalized epilepsy; SVP, sodium valproate.

(33.4\%) had intermittent relapses due to various reasons, but $53(14.4 \%)$ could still be managed with doses $<1,000 \mathrm{mg} /$ day (-Table 2). When variable compared between the groups of patients who get relapsed versus not relapsed showed that longer duration of illness $(p=0.01)$, initial poor control of seizures $(p=0.001)$, number, and high doses of antiepileptic medications $(p=0.000)$ were associated with significantly high chances of relapse.

\section{Discussion}

IGE is found in 21\% patients of all patients presenting with seizures. The IGE is found in one-fourth to one-fifth proportions of all seizures patients. ${ }^{14,15}$ In our cohort, IGE was the third commonest after granuloma related acute symptomatic seizures and cryptogenic epilepsy. ${ }^{16}$ The male to female ratio was variable in different studies from India, equal male to female ratio in one, ${ }^{17}$ female predominate in other ${ }^{2}$ and male predominate in ours, and other study from southern India. ${ }^{18}$

IGE is a medical and social problem for young generation as most of the patients had onset before age of 20 years. ${ }^{2}$ At this age personality changes, carrier planning and marriage planning are important life related issues at the age between 18 and 25 years. ${ }^{19}$ Person suddenly starting symptoms of 
Table 2 Showing number of patients having successful treatment with low-dose sodium valproate

\begin{tabular}{|c|c|c|c|}
\hline Treatment group $(n)$ & $\begin{array}{l}\text { Number (\%) on low-dose } \\
(<1,000 \mathrm{mg} / \text { day) SVP }\end{array}$ & $\begin{array}{l}\text { Number }(\%) \text { on high } \\
\text { dose (>1,000 mg/day) } \\
\text { SVP due to relapse on } \\
\text { low doses in follow-up }\end{array}$ & $\begin{array}{l}\text { Number (\%) had relapse } \\
\text { during follow-up but } \\
\text { continued on low-dose SVP } \\
(<1,000 \mathrm{mg})\end{array}$ \\
\hline On SVP monotherapy $(n=155)$ & $129(83.2)$ & \multirow[t]{4}{*}{$26(16.8)$} & \multirow[t]{4}{*}{$13(8.4)$} \\
\hline 200-300 mg/day & $22(17)$ & & \\
\hline 400-600 mg/day & $81(62.8)$ & & \\
\hline 700-900 mg/day & $26(20.2)$ & & \\
\hline On SVP + LTG $(n=127)$ & $100(78.6)$ & \multirow[t]{4}{*}{$27(21.4)$} & \multirow[t]{4}{*}{$18(14.2)$} \\
\hline 200-300 mg/day & $15(15)$ & & \\
\hline $400-600 \mathrm{mg} /$ day & $64(64)$ & & \\
\hline 700-900 mg/day & $21(21)$ & & \\
\hline On SVP + CZP or SVP + LTG + CZP $(n=86)$ & $69(80)$ & \multirow[t]{4}{*}{$17(20)$} & \multirow[t]{4}{*}{$22(25.6)$} \\
\hline 200-300 mg/day & $7(8.1)$ & & \\
\hline 400-600 mg/day & $39(45.3)$ & & \\
\hline 700-900 mg/day & $23(26.6)$ & & \\
\hline Overall $(n=368)$ & $298(81)$ & $70(19)$ & $53(14.4)$ \\
\hline
\end{tabular}

Abbreviations: CZP, clonazepam; LTG, lamotrigine; SVP, sodium valproate.

disease like IGE that affects their whole life style is commonly very disturbing for the young generation. Therefore, the treatment planning does not mean drug treatment only, but needs holistic management and counseling of person suffering from IGE and family.

Little less than half of our patients presented with poor control of seizures. The issue of wrong selection of antiepileptic drugs has been raised by many studies in the past. ${ }^{17,18}$ The patients with IGE often presents with pseudo-refractory epilepsy due to wrong selection of drugs and correct drug selection results in seizures remission. ${ }^{20,21}$ Of 187 patients presenting with poor control due to wrong selection of drugs, $146(78 \%)$ had full remission after adequate treatment. Thus, adequate drug selection and proper counseling of person with IGE is key for better seizure control and quality of life in affected young and productive age group.

Guidelines for proper drug selection for GTCS in patient with IGE are in favor of SVP, LEV, or LTG. For myoclonic jerks, clonazepam (CZP) and for absence seizures, ethosuximide (ETM) are specific drugs available in Indian market. Head-to-head drug trial on monotherapy in IGE were in favor of SVP.,22 In two studies comparing SVP with LTG showed that SVP is far better choice over LTG in generalized epilepsy. ${ }^{6,7}$ In SANAD II (unpublished), data presented in 33rd International epilepsy congress 2019, showed that SVP was better than LEV in all aspects. Even SVP is the proven as the best monotherapy in IGE patients, but lots of concerns are taken for its usage in patients with epilepsy (especially, female patients). ${ }^{9}$ Main concern was valproate associated teratogenicity in child-bearing age groups female. There are specific guidelines available for using valproate in girls and in female with child-bearing potentials. ${ }^{23}$ The issues of risk associated with valproate in pregnancy were so prominent that valproate was replaced by other antiepileptic medications, which were less risky during pregnancy but also less effective. Later, it was suggested that risk of seizures-related complications in pregnancy are as prominent as drug-related problems, and it was added that female well controlled on valproate can continue to take same drug during pregnancy. Additional use of folic acid prior to pregnancy and lower doses ( $<700 \mathrm{mg}$ ) were associated with lesser chances of congenital malformations. ${ }^{23}$

The young patients suffering from IGE have many questions related to their future living with disease and drugs. Major concern is obviously drug-related side effects, especially in girls and female of child-bearing age. When the option of low dose and effective treatment with SVP is available to patients, it could be a good consolation point to relieve their anxiety. ${ }^{11}$ We named the initial phase of "intensive phase" of valproate therapy (high dose) for 1 year followed by, "tapering phase" in second year, and lastly "maintenance phase" (low dose) for rest of the life. There have been reports that primary physicians some time opine to the patients with IGE that they can stop antiepileptic drugs after 3 to 5 years of successful remission. One study showed that $95.4 \%$ had seizures relapse with 24 months after antiepileptic withdrawal in patients with IGE. ${ }^{24}$ In our cohort, $21.5 \%$ patients presented with relapse of seizures after drug withdrawal that further complicate the situation as many patients suffer from grievous injuries and mental trauma as a consequence of unpredicted seizures after so called "full course of treatment" of 3 to 5 years. Telling truth to the patients in the beginning of treatment with correct diagnosis of IGE and correct drug selection is a better option rather than wrong information and wrong treatment. Findings from our study favor the point that low-dose valproate therapy in patients with IGE 
is a good option to prevent seizure relapse and also to minimize the long-term drug-related side effects. With the option of low-dose valproate therapy, we should not deprive our patients with IGE from the most effective treatment available at least in current basket of antiepileptic drugs.

Another option for IGE patients having effective and low-risk treatment is combination of valproic acid and LTG. Combination of valproate with LTG has advantage of synergistic effect and longer half-life when taken as single bed time dose..$^{25}$ Maintaining low-dose SVP with synergistic LTG will be helpful to reduce long-term and pregnancy-related side effects of valproic acid with little higher acute risk of coetaneous reaction. ${ }^{26}$ It has been shown that SVP and LTG used separately as monotherapy in high doses had poor control of seizure as compared with combination of both in lower doses (VPA + LTG). ${ }^{27}$ We could successfully treat many patients with low-dose combination of SVP and LTG with low adverse reactions and high efficacy. Valproate and CZP combination also had shown to be effective in patients with intractable myoclonic epilepsy. ${ }^{28}$

\section{Conclusion}

To conclude, there is plenty of evidence that SVP is still have good support for considering it a drug of choice for persons with IGE. The concern of side effects associated with long-term use of SVP can be minimized with low-dose maintenance therapy of SVP with or without LTG or CZP.

\section{Conflict of Interest}

None declared.

\section{References}

1 Douglas RN. Idiopathic generalized epilepsy recognised by the International League Against Epilepsy. Epilepsia 2005; 46:48-56

2 Sinha S, Pramod MN, Dilipkumar S, Satishchandra P. Idiopathic generalized epilepsy: phenotypic and electroencephalographic observations in a large cohort from South India. Ann Indian Acad Neurol 2013;16(2):163-168

3 Beydoun A, D'Souza J. Treatment of idiopathic generalized epilepsy: a review of the evidence. Expert Opin Pharmacother 2012;13(9):1283-1298

4 Martinovic Z. Treatment and prognosis of idiopathic generalized epilepsy in adults. Clin Neurophysiol 2015;9:e176-e184

5 Marson AG, Al-Kharusi AM, Alwaidh M, et al; SANAD Study group. The SANAD study of effectiveness of valproate, lamotrigine, or topiramate for generalised and unclassifiable epilepsy: an unblinded randomised controlled trial. Lancet 2007;369(9566):1016-1026

6 Mazurkiewicz-Bełdzińska M, Szmuda M, Matheisel A. Long-term efficacy of valproate versus lamotrigine in treatment of idiopathic generalized epilepsies in children and adolescents. Seizure 2010;19(3):195-197

7 Tang L, Ge L, Wu W, et al. Lamotrigine versus valproic acid monotherapy for generalised epilepsy: a meta-analysis of comparative studies. Seizure 2017;51:95-101

8 Villanueva V, Montoya J, Castillo A, et al. Perampanel in routine clinical use in idiopathic generalized epilepsy: the 12-month GENERAL study. Epilepsia 2018;59(9):1740-1752
9 Tomson T, Marson A, Boon P, et al. Valproate in the treatment of epilepsy in girls and women of childbearing potential. Epilepsia 2015;56(7):1006-1019

10 Tomson T, Battino D, Bonizzoni E, et al; EURAP Study Group. Dose-dependent teratogenicity of valproate in mono- and polytherapy: an observational study. Neurology 2015;85(10): 866-872

11 Miró J, Aiguabella M, Veciana M, et al. Low-dose sodium valproate in the treatment of idiopathic generalized epilepsies. Acta Neurol Scand 2014;129(5):e20-e23

12 From the Commission on Classification and Terminology of the International League Against Epilepsy. Proposal for revised clinical and electroencephalographic classification of epileptic seizures. Epilepsia 1981;22(4):489-501

13 Proposal for revised classification of epilepsy and epileptic syndrome. Commission on classification and terminology of the International League Against Epilepsy. Epilepsia 1989;30:389-399

14 Jallon P, Latour P. Epidemiology of idiopathic generalized epilepsies. Epilepsia 2005;46(Suppl 9) :10-14

15 Gastaut H, Gastaut JL, Gonçalves e Silva GE, Fernandez Sanchez GR. Relative frequency of different types of epilepsy: a study employing the classification of the International League Against Epilepsy. Epilepsia 1975;16(3):457-461

16 Goel D, Mittal M. Mono-therapy versus poly-therapy: ten years Indian experience on various seizure disorders. World J Neurosci 2015;5:350-357

17 Jha S. Juvenile myoclonic epilepsy in India. Some interesting observations. Ann Indian Acad Neurol 2004;7:417-420

18 Vijai J, Cherian PJ, Stlaja PN, Anand A, Radhakrishnan K. Clinical characteristics of a South Indian cohort of juvenile myoclonic epilepsy probands. Seizure 2003;12(7):490-496

19 Singh G, Pauranik A, Menon B, et al. The dilemma of arranged marriages in people with epilepsy. An expert group appraisal. Epilepsy Behav 2016;61:242-247

20 Alsaadi T, Taha H, Al Hammadi F. Choice of antiepileptic drugs in idiopathic generalized epilepsy: UAE experience. Epilepsy Res Treat 2015;2015:184928

21 Benbadis SR, Tatum WO IV, Gieron M. Idiopathic generalized epilepsy and choice of antiepileptic drugs. Neurology 2003; 61(12):1793-1795

22 Steinhoff BJ, Ueberall MA, Siemes H, Kurlemann G, Schmitz B, Bergmann L; LAM-SAFE Study Group. The LAM-SAFE Study: lamotrigine versus carbamazepine or valproic acid in newly diagnosed focal and generalised epilepsies in adolescents and adults. Seizure 2005;14(8):597-605

23 Thomas SV, Valproic acid use in girls and women with epilepsy. In: Mehndiratta MM, ed. Valproic Acid: Indian Consensus Document by Indian Epilepsy Society. Elsevier 2016 ;54-58

24 Pavlović M, Jović N, Pekmezović T. Antiepileptic drugs withdrawal in patients with idiopathic generalized epilepsy. Seizure 2011;20(7):520-525

25 Kanner AM, Frey M. Adding valproate to lamotrigine: a study of their pharmacokinetic interaction. Neurology 2000; 55(4):588-591

26 Ghaffapour M, Hejazie SS, Harirchian MH, Pourmahmoodian H. Phenytoin, Carbamazepine, Sodium Valproate and Lamotrigine induced cutaneous reactions. Acta Med Iran 2005;43:37-42

27 Moeller JJ, Rahey SR, Sadler RM. Lamotrigine-valproic acid combination therapy for medically refractory epilepsy. Epilepsia 2009;50(3):475-479

28 Mirelesh R, Leppik IE. Valproate and clonazepam co medication in patients with intractable epilepsy. Epilepsy 1985;26: 122-126 